\title{
Business Model Comparison Between Spotify and NetEase Cloud Music
}

\author{
Yonghao $\mathrm{Li}^{1, *}$, Qirui Zhang ${ }^{2}$, Anna Rita $\mathrm{Li}^{3}$, Jun Cheng ${ }^{4}$ \\ ${ }^{1}$ Battle Ground Academy, Franklin, Tennessee, 37069, USA. \\ ${ }^{2}$ Battle Ground Academy, Franklin, Tennessee, 37069, USA \\ ${ }^{3}$ Shanghai Gold Apple School: 1555 Jufeng Road, Pudong New Area, 200120 \\ ${ }^{4}$ Peninsula Catholic High School, Newport News, Virginia, 23601, USA \\ *yonghaoli22@mybga.org
}

\begin{abstract}
"With the development of technologies, the importance of music has been increased. Based on one of the researches about music, it pointed out that music somehow has the ability to release our pain, help release our stress or give us confidence. In one sentence, music has a powerful therapeutic effect on the human psyche". In the past of the music market, the control was generally dispersed and hard to be gathered together. However, now the market of music in China is mainly dominating by NetEase Cloud Music while Spotify owns a lot of markets in the world range. The main focus of this article will be illustrating the business model for both companies and put together for comparison to get a conclusion about if the business model works for both countries or not.
\end{abstract}

Keywords: Spotify, NetEase Cloud Music, Freemium

\section{INTRODUCTION}

\subsection{Importance of music in human life}

Music plays an important role in each of our lives. Music is the universal language of mankind, the ubiquitous source of entertainment, the medium that changes our emotions, and the medium that elevates us to higher emotions and experiences that affect all levels of human existence - physical, mental, and spiritual. Music does not speak specific words, it speaks emotionally.

\subsection{Freemium}

The term "freemium" is a combination of the words "free" and "premium". Companies will be based on different customer groups and charge differently. The way this business model works is by separating the customer groups into paid users and free users. All these free users, obviously, they have lots of limitations as they are not paying for anything. All the reason company set up the business model is to hope these customers will willing to upgrade to premium.

Both companies going to do the comparison today are all following the "freemium" business model.

\subsection{Spotify}

1.3.1 Why now: Because of the increasing piracy problem in music industry

1.3.2 Ideal Customers: Millennials take most of the percentage of Spotify users. (• Spotify Users by Age in the U.S. 2018 | Statista, n.d.) [1]

\subsubsection{LTV}

1.3.3.1 ARPU: Average subscription fee Spotify charges against users (5.4 dollars)

1.3.3.2 CRPU: Average spend on supporting the product (3.9 dollars)

1.3.3.4 Churn Rate: The number of customer lost in a certain period of time. $(4.5 \%)$

\subsubsection{Lifetime Value}

Lifetime Value 
Lifetime Value $=\frac{5.4 \text { dollars }-3.9 \text { dollars }}{0.045}$

$$
=33 \text { dollars }
$$

1.3.4 Competitive Advantage: Able to increase the engagement on based its massive amount of listener data and its ability to leverage that data.

\subsection{Free User vs. Paid User (Spotify)}

Since 2015, the number of Spotify users is increasing exponentially while the subscribe user is also increasing exponentially and is in proportion to the overall users. However, it is obvious that compare to pay for the premium, most of the user more willing to experience the free mode.

\section{MAIN FOCUS AND DISCUSSION}

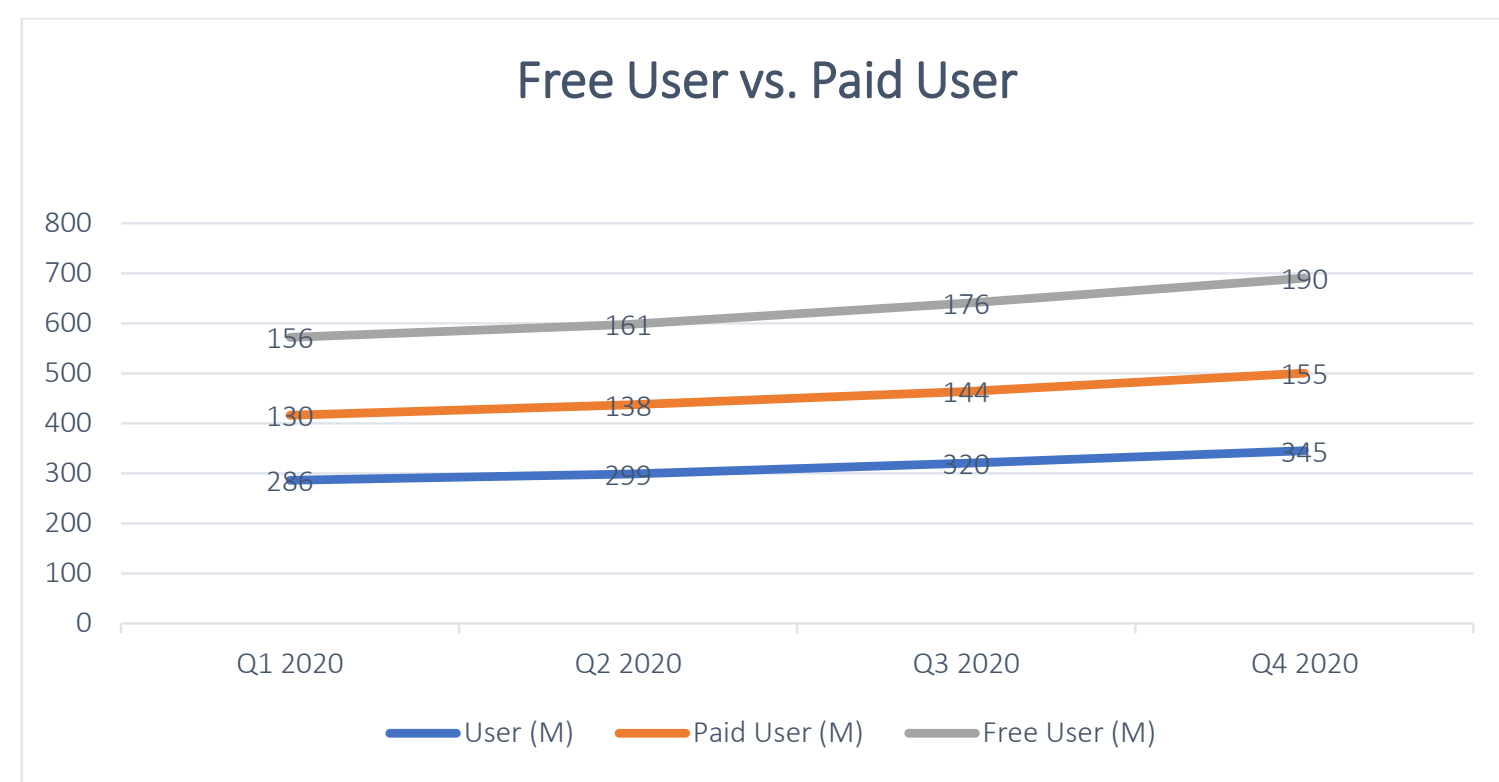

Figure 1 - Free Users vs. Paid Users

\subsection{What are they paying for?}

- Unlimited high-qualities streaming access over 50 million songs

- Travel abroad with no limitation
- Ad free music

- Unlimited skips

Ranking of media companies in Sweden in 2019, by revenue

In 2019 Sweden, Spotify made 7.2 billion (Swedish Krona) through the year which is around 7 billion (Euro). 


\section{Ranking of media companies in Sweden in 2019, by revenue} (in million SEK)

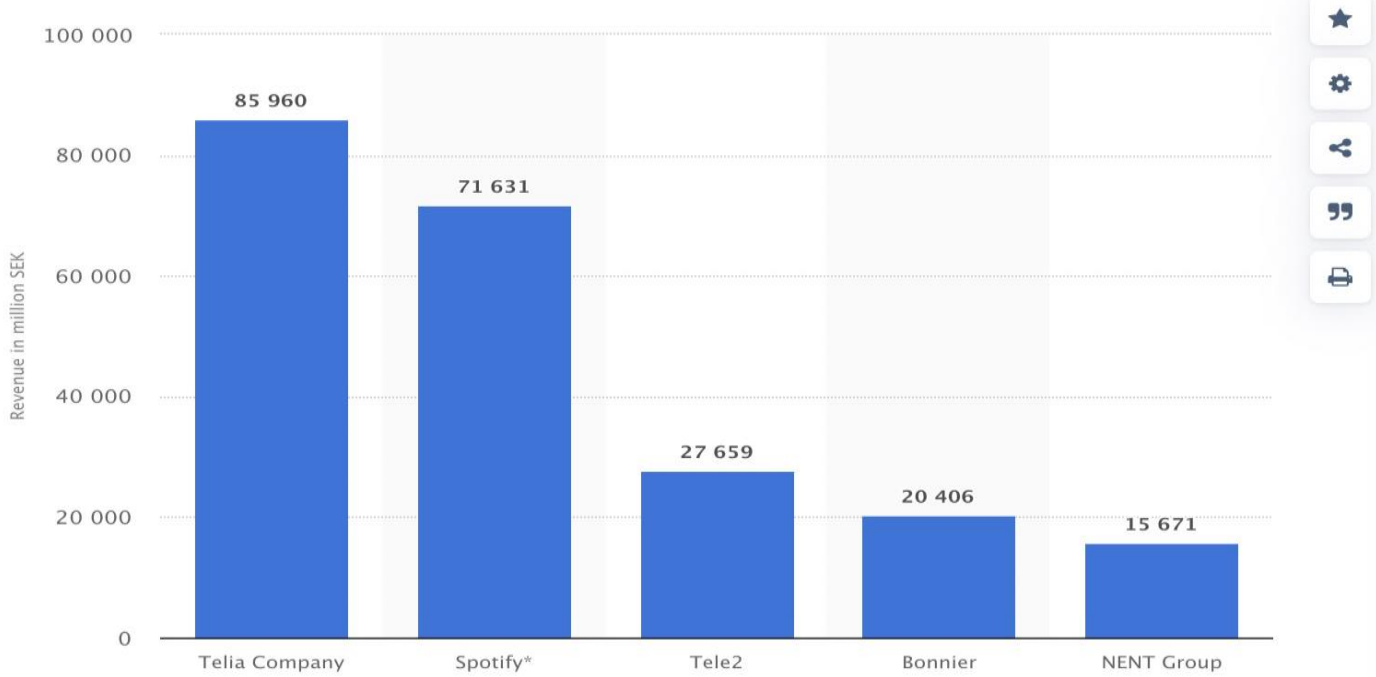

\subsection{Business Model}

\subsubsection{Freemium}

Spotify operates under a "freemium business model (basic services are free, while additional features are provided through paid subscriptions). Spotify makes money through selling albums and advertising to other platforms." (editor et al., n.d.) [2]

Spotify reaches the companies and artists for their music and copyrights by paying the price with royaltiesand $70 \%$ of the total goes to the right holder. The amount of money artists earn are based on different foctors such as country or roalty rate and usually the average received by at last is between US $\$ 0.000029$ and US\$0.0084.

"Spotify offers unlimited subscription packages, close to the economic equilibrium of the recording industry estimated by the Open Music Model (OMM). However, the inclusion of digital rights management (DRM) restrictions is different from OMM and competitors, such as iTunes Store and Amazon Music, which are abandoning DRM" ("Spotify," 2021). [3]

\subsubsection{Spotify Premium (Spotify Premium, n.d.)} [4]:

Individual: 9.99 dollars / month (1 account)

Duo: 12.99 dollars / month (2 accounts)

Family: 15.99 dollars / month (6 accounts)

Student: 4.99 dollars / month (1 account)

\subsubsection{Free Version:}

Shuffle-only mode

Limited amount of skips / hour (6 skips)

Spotify Radio not Available

\begin{tabular}{|c|c|c|c|c|c|}
\hline \multicolumn{7}{|c|}{ Spotify annual costs by area, EUR millions } \\
\hline Area & 2016 & 2017 & 2018 & 2019 & 2020 \\
\hline Sales and marketing & 368 & 567 & 620 & 826 & 1029 \\
\hline R\&D & 207 & 396 & 439 & 615 & 837 \\
\hline General and administrative & 175 & 264 & 283 & 352 & 442 \\
\hline Total & 750 & 1200 & 1400 & 1800 & 2300 \\
\hline
\end{tabular}

Figure 3 - Free version vs. Premium referring function wise 


\subsection{SWOT Model for Spotify}

\section{Strengths}

1. High popularity

2. Easy accessibility through different platforms[5] (Brandon Gaille, 2021)

\section{Weaknesses}

1. Could not make the deal with the rights holder over the price

2. Dependence on technology companies including its competitor

4. Imitable business model

\section{Opportunities}

1. Potential market based on the new form of music

2. Diversification based on customer segments

3. Looking for new partnerships besides those who already exist

\section{Threats}

1. Competition with the companies exist in the market

\section{Legislature articles} [6]

Royalties (Help - Royalties - Spotify for Artists, n.d.)

Music on Spotify earns two kinds of royalties

\section{Recording royalties}

When Spotify gains the profit from all the major activities, these incomes will go through the licensors to the artists who cooperated with the platform to publishe their songs.

\section{Publishing royalties}

The publishers of Spotify have the responsiblity to communicate with the collecting societies and mechanical agencies who also AKA third party companies and these they are playing the role of distributing the income to the song writers and owners. When a song gets played on Spotify, the rightsholders receive royalties for it, whether it's played by a Premium or ad-supported customer.

\subsection{Data gathering cost}

\section{Spotify annual costs by area, EUR millions}

$\begin{array}{cccccc}\text { Area } & 2016 & 2017 & 2018 & 2019 & 2020 \\ \text { Sales and marketing } & 368 & 567 & 620 & 826 & 1029 \\ \text { R\&D } & 207 & 396 & 493 & 615 & 837 \\ \text { General and administrative } & 175 & 264 & 283 & 354 & 442 \\ \text { Total } & 750 & 1200 & 1400 & 1800 & 2300\end{array}$

Figure 4: Spotify annual costs by area, EUR millions Source: Spotify (Spotify — Financials, n.d.)[7]

Spotify has spent 837 EUR millions on data gathering.
NetEase Cloud Music has spent 576 million RMB on the data gathering cost. 


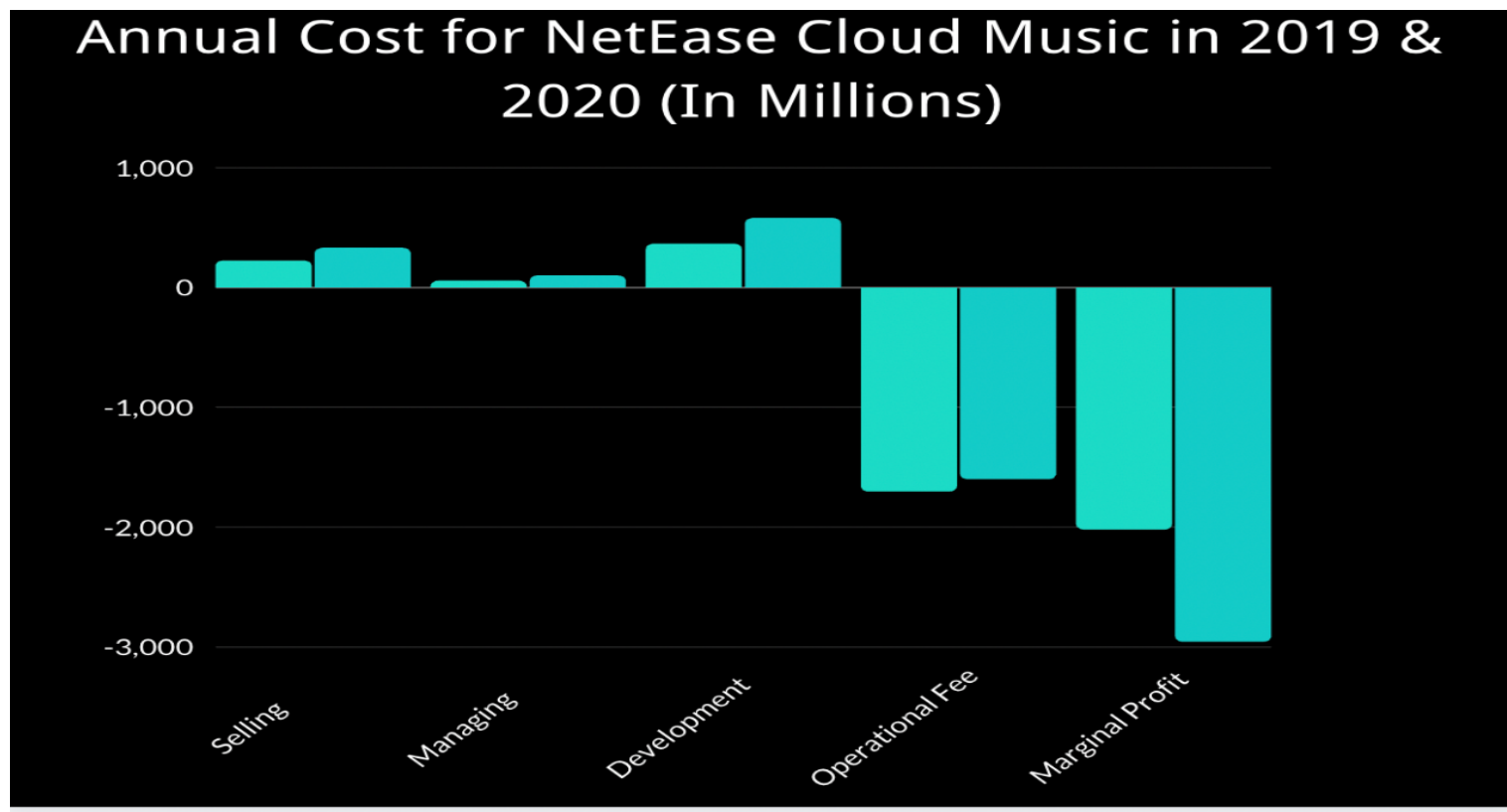

Figure 5 - NetEase Music annual costs by area, CNY

\subsection{NetEase Cloud Music}

\subsubsection{Overall introduction (NetEase Cloud Music)}

\subsubsection{Ideal Customers}

Females (Percentage increased from $14 \%$ to $26 \%$ )

Groups aged below 30 (Over 86\% of overall customers)

Students (Over half of them are students)

\subsubsection{Funding}

NetEase (Mother company)

Alibaba (Strategic Partnership)

Baidu (Strategic Partnership)

\subsubsection{Competitive Advantages: NetEase has the revenue to purchase copyrights which provides customers more options}

\section{Brief Introduction}

The release of the "Strict Copyright Order" in 2015 marked the official entry of the Chinese digital music market into the era of legalization. NetEase Cloud Music, which was only established in 2013, is doing a differentiated layout. It has been a step slower in copyright snapping, losing to the well-capitalized Tencent and Alibaba products, almost becoming the only loser. In recent years, NetEase Cloud Music has been trying to get rid of its copyright ties. Among them, the one that caused the most heated discussion was that on
April 1, 2018, NetEase Cloud Music suddenly removed the songs of Chinese famous singer Jay Chou. It is understood that the loss of the copyright competition directly led to the loss of a huge number of customer base of NetEase Cloud Music.

\subsection{Regulation (All requirements are based on the proposal from the government of the Republic of China)}

\subsubsection{Anti-Addiction Mode}

Whenever a kid is registering an account for certain apps, they have to provide valid identity information to get everything all set. However, some "smart" kids would memorize their parents' identification number so they could reach their goals. Further in 2019, the National Cyberspace Administration of China evaluated the antiaddiction effects of the 20 online video platforms that had previously launched the "Youth Mode", guided relevant platforms to adjust and optimize their function settings, and completely cleared out the exclusive content pool for teenagers. "On the basis of summarizing the previous experience of promotion, these 53 online "youth mode" network platforms have realized a unified operation mode and unified functional standards. In this mode, site search, bullet screen comments, content sharing, private message chat, shooting release, Features such as recharge and rewards only recommend content suitable for teenagers to watch, ensuring that the content pool under the "teenage mode" is healthier and more beneficial." (Resolutely Curb Addiction and Protect the healthy growth of young people -- An official of the National Press and Publication Administration answers reporters questions on the Notice on Preventing Minors from Indulging in Online Games.) [8] 


\subsubsection{Urban Language Management System}

China is a country with thousands of years of history. During the development, there were so many urban languages which are so-called bad words nowadays got create. Since China is developing rapidly, the government was proposing to control our word usage so the cities or provinces can actually turn out with a better appearance. Due to this reason, NetEase came out with control to the explicit contents which mean when you are playing music that includes any form of bad words, users are able to skip the song or using asterisks to cover the bad words shown in the lyrics. Especially nowadays elementary school students will learn more about language civilization and the importance of civilization through class. "The teachers hope that through the classroom, students will have a good etiquette literacy and deepen their understanding of language civilization etiquette. Constrain students' behaviors by discovering uncivilized behaviors around them." (Urban Language Management and Construction of Urban Language Civilization - China Social Sciences Network, N.D.) [9]

\subsubsection{In compared to Spotify}

Due to the different background cultures, United States does not care about bad words in songs as much as in China. As a 17-year-old kid, Spotify does not provide any where showing that I have the control to get rid of these songs which includes bad words.

\subsection{SWOT Model for NetEase Cloud Music}

\section{Strength}

1. UI (user interface) Design

2. Music Community for comments and interactions

3. Algorithmic Recommendations and Personalization

4. Special Pages of branded Signers \& Music Producers

5. Development Plan of Original Music Producers

6. Tickets for Music Festival \& Concerts

\section{Weakness}

1. Insufficient music and songs of copyrights

2. Unclear Music Genre Division

3. Limitation in Access to song listening and downloading outside Mainland China

\section{Opportunity}

1. Improvement in awareness of music copyright protection

2. Establishment of music interaction and socialization

3. Limitation in access to song listening and downloading outside Mainland China

4. Convenience provided by increase in Mobile Terminals

5. Purchase of online listening rights without data consumption

\section{Threat}

1. Lack of capitals in music copyright purchase

2. Lack of corporate alliances

3. Management staff change

\section{SOURCES OF INCOME}

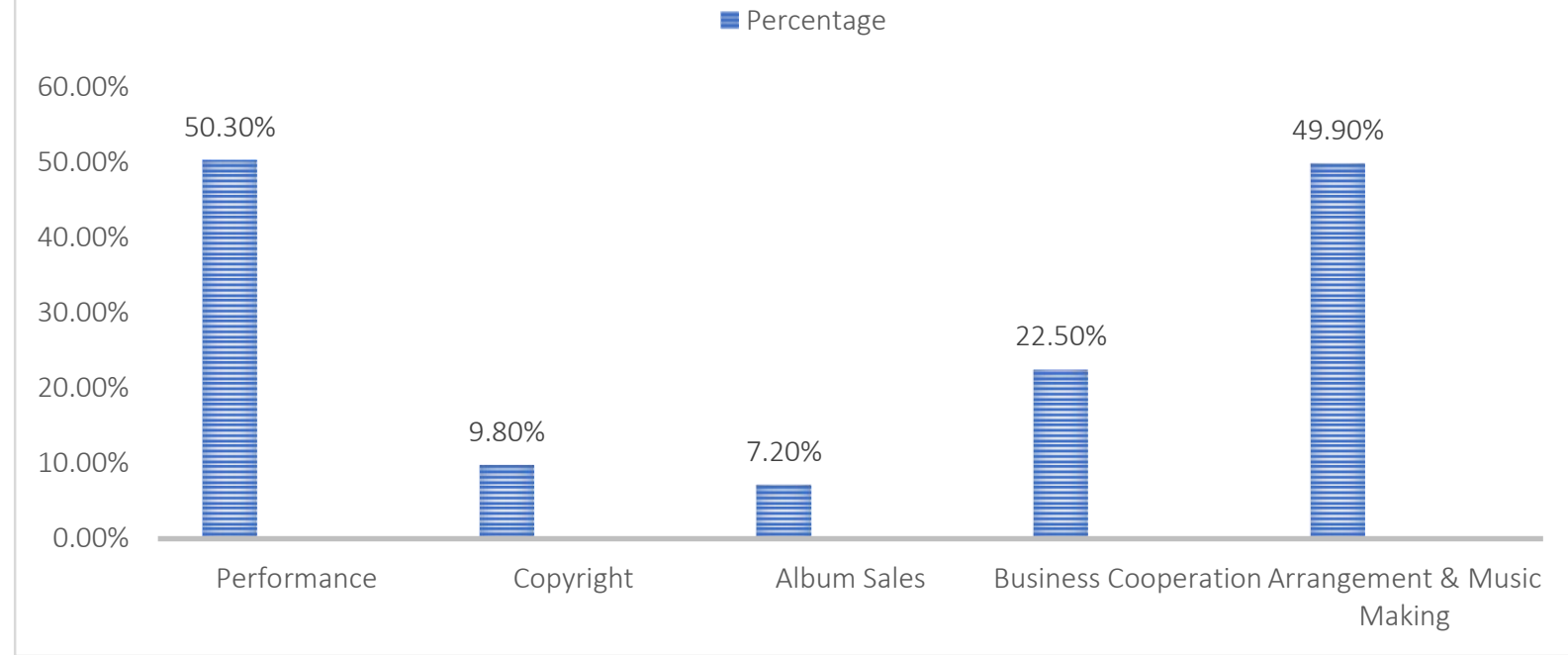

Figure 6 - Sources of Income 


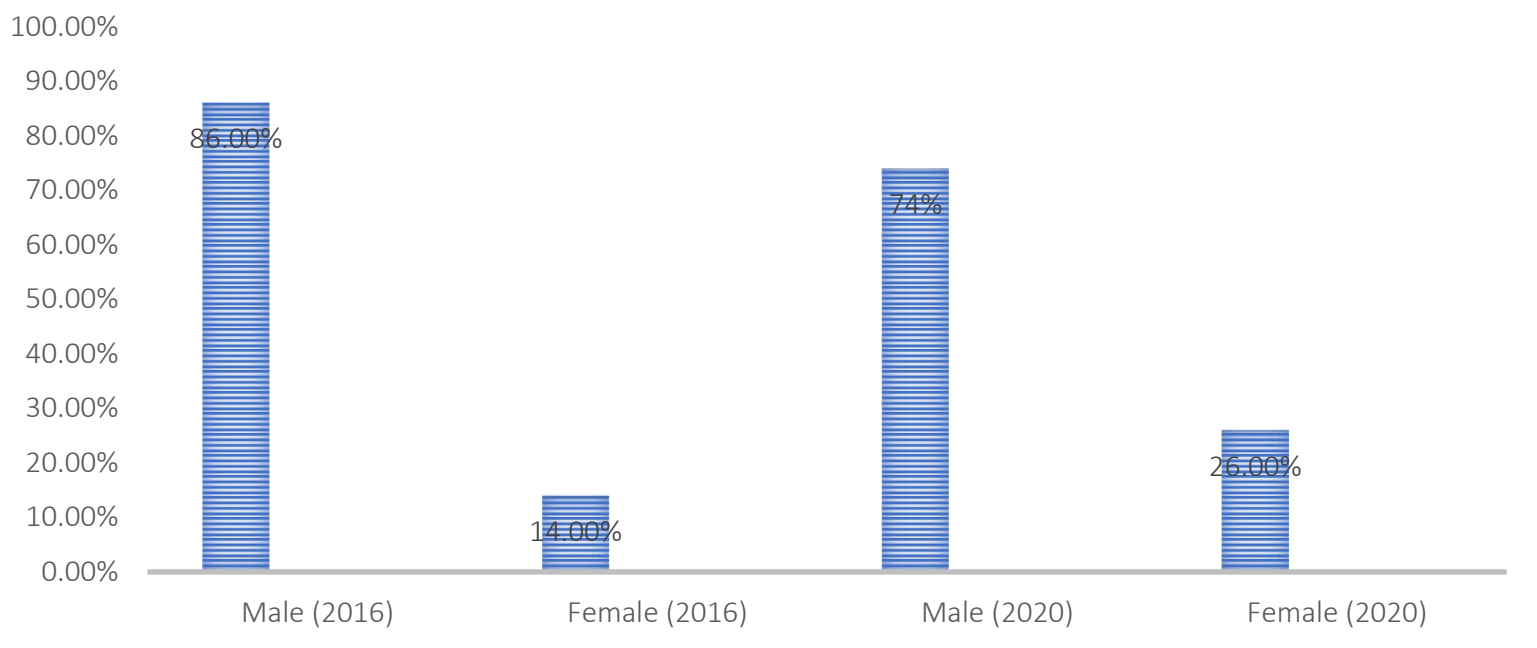

Figure 7 - Percentage of Musicians in 2016 and 2020 (By Gender)

Sources of Income \& Average Income per musician

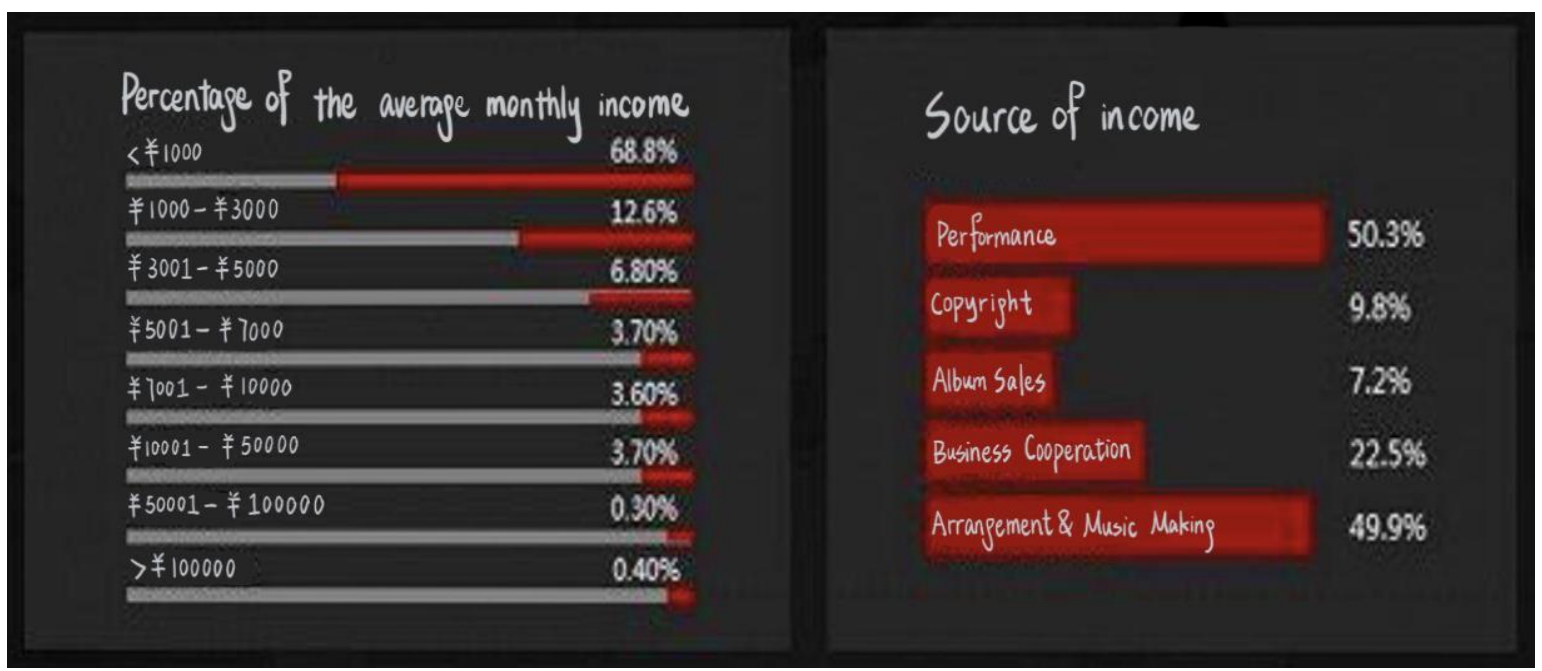

Figure 8 - Source of income (left) \& Average income per musician (right)

\subsection{Musician Income \& Source of income}

As the left side of the figure 7 shows, the majority of the artists / musicians earn less than 1000 per month which means they did not get what they deserve. On the other side, there is a group of artists / musicians who are famous, welcome, and have some well-known music, they are standing on the top of the pyramid of the music industry by making fifty thousand to a hundred thousand per month. To the right side of the figure, it is a chart showing the structure of income sources. Obviously, the top two are performance and music making and arrangement. Literally, these are the two can provide them with the maximum benefits. This phenomenon actually has been exist for the past years. On one hand, the threshold of musicians is getting lower and lower; on the other hand, the income of music copyright is becoming more and more bipolar, which leads to the low income of musicians and increased income difference among individuals. Industry insiders believe that the status quo is likely to change if the use of music copyright is regulated and royalties are substantially increased. The premise is that the work is good, the musicians are good, and the platform supports it. , n.d.) [10]

\section{CONCLUSION}

Spotify has a mature operating system. They have expanded the scale worldwide as shown in Figure 1. Spotify already covers more than 150 countries and 6 different continents. They never only focus on the present moment, instead, they more care about long-term benefits. Since they founded, they made up the business model and keep up with it. They rather give up some present interests to obtain long-term development. 
NetEase once shortly gained lots of customers in the market and suddenly turn down and not able to climb back in years. They do not have a good reputation in the market as they would suddenly remove songs without any announcement or have a decreasing number of copyrights of songs. Once someone commented that if QQ music could do better on matching up the lyrics and the music, NetEase music would not be their first option. As the mother company, NetEase has to figure out ways to improves its functions or services so they can avoid customer losing to keep themselves competitive in the market.

\section{REFERENCES}

[1] Spotify users by age in the U.S. 2018 | Statista. (n.d.). Retrieved July 15, 2021, from https://www.statista.com/statistics/475821/spotifyusers-age-usa/

[2] Brandon Gaille. (2021, January 11). Spotify SWOT Analysis for 2021: 26 Strengths and Weaknesses. BrandonGaille.Com.

https://brandongaille.com/spotify-swot-analysis/

[3] editor, F. B. F. L. F. T. T. S. is an, finance, writer S. has 20+ years of experience covering personal, Management, W., \& Segal, business news L. about our editorial [4] policies T. (n.d.). What You Should Know About Freemiums. Investopedia. Retrieved July 12, 2021, from https://www.investopedia.com/terms/f/freemium.as $\mathrm{p}$

[4] Help-Royalties - Spotify for Artists. (n.d.). Retrieved July 15, 2021, from https://artists.spotify.com/help/article/royalties, /help/article/royalties
[5] Spotify. (2021). In Wikipedia. https://en.wikipedia.org/w/index.php?title=Spotify \&oldid=1033753021

[6] Spotify Premium. (n.d.). Spotify. Retrieved August 6, 2021, from http://www.spotify.com/us/premium/

[7]Spotify-Financials. (n.d.). Retrieved August 6, 2021, from

https://investors.spotify.com/financials/default.aspx

[8] Xinhua News Agency. (2019, November 5). [Resolutely curb addiction and protect the healthy growth of young people-the relevant person in charge of the National Press and Publication Administration answered reporters' questions on the "Notice on Preventing Minors from Indulging in Online Games"]. China News Agency. Retrieved September 30, 2021, from file://Users/liyonghao/Zotero/storage/EYGYNA4 D/content_5448848.html

[9] China Social Science Web. (2020, August 4). [Urban Language Management and Construction of Urban Language Civilization]. WWW. CSSN.CN.

Retrieved September 30, 2021, from http://www.cssn.cn/yyx/202008/t20200804_51654 47.shtml

[10] Beijing Youth Newspaper. (2021, January 10). [How to make money? $52 \%$ of musicians have no music income, and copyright income is polarized]. www.chinanews.com. Retrieved September 30, 2021,

from file:///Users/liyonghao/Zotero/storage/LFMY4YS2/ 9383073.html 